\title{
BMJ Open Protocol for a systematic review of the effects of interventions to inform or educate caregivers about childhood vaccination in low and middle-income countries
}

\author{
Lungeni A Lukusa, ${ }^{1}$ Nyanyiwe N Mbeye, ${ }^{2}$ Folasade B Adeniyi, ${ }^{2}$ \\ Charles S Wiysonge ${ }^{1,2,3}$
}

To cite: Lukusa LA, Mbeye NN, Adeniyi FB, et al. Protocol for a systematic review of the effects of interventions to inform or educate caregivers about childhood vaccination in low and middle-income countries. BMJ Open 2015;5:e008113. doi:10.1136/bmjopen-2015008113

- Prepublication history and additional material for available. To view please visit the journal (http://dx.doi.org/ 10.1136/bmjopen-2015008113)

Received 5 March 2015 Revised 21 May 2015 Accepted 15 June 2015

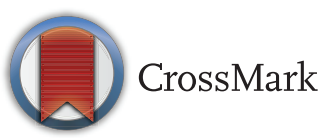

\footnotetext{
${ }^{1}$ Division of Community Health, Faculty of Medicine and Health Sciences, Stellenbosch University, Cape Town, South Africa

${ }^{2}$ Centre for Evidence-based Health Care, Faculty of Medicine and Health Sciences, Stellenbosch University, Cape Town, South Africa

${ }^{3}$ Cochrane South Africa, South African Medical Research Council, Cape Town, South Africa
}

Correspondence to Dr Lungeni A Lukusa; augustolungeni@yahoo.com

\section{ABSTRACT}

Introduction: Despite their proven effectiveness in reducing childhood infectious diseases, the uptake of vaccines remains suboptimal in low and middleincome countries. Identifying strategies for transmitting accurate vaccine information to caregivers would boost childhood vaccination coverage in these countries. The purpose of this review is to assess the effects on childhood vaccination coverage of interventions for informing or educating caregivers about the importance of vaccines in low and middle-income countries, as defined by the World Bank.

Methods and analysis: Eligible study designs include randomised controlled trials (RCTs) as well as non-randomised controlled trials (non-RCTs). We will conduct a comprehensive search of both peer-reviewed and grey literature available up to 31 May 2015. We will search PubMed, Scopus, Cochrane Central Register of Controlled Trials, Web of Science, Cumulative Index of Nursing and Allied Health, prospective trial registries and reference lists of relevant publications. Two authors will independently screen the search output, retrieve full texts of potentially eligible studies and assess the latter against predefined inclusion criteria. Disagreements between the two authors will be resolved through consensus and arbitration by a third author. We will pool data from studies with homogenous interventions and outcomes, using random-effects meta-analysis. We will assess statistical heterogeneity using the $\chi^{2}$ test of homogeneity (with significance defined at the $10 \% \alpha$-level) and quantify it using Higgins' inconsistency index. We will explore the cause of any observed statistical heterogeneity using subgroup analysis, with subgroups defined by study design (RCTs vs non-RCTs) and type of intervention (information vs educational interventions).

Ethics and dissemination: The proposed systematic review will collect and analyse secondary data that are not associated with individuals. The review will make a significant contribution to the knowledge base of interventions for improving childhood vaccination coverage in low and middle-income countries.

\section{Strengths and limitations of this study}

- This study will contribute to strengthen the evidence base on effective interventions for improving immunisation coverage in resourceconstrained settings.

- We will use the GRADE system to ascertain the strength of the evidence base for each outcome and report data for the primary outcome in a 'Summary of Findings' table.

- We have written the protocol following the recently published PRISMA-P guidelines.

- We will include non-randomised trials, which are prone to have a high risk of bias and are likely to produce evidence of low certainty. To mitigate this risk, we plan to conduct sensitivity analyses to assess the robustness of the findings to risk of bias by excluding studies with a high risk of bias.

Protocol registration number: PROSPERO, CRD42014010141.

\section{INTRODUCTION}

The use of vaccines during childhood has been one of the most effective public health interventions for combating infectious diseases. ${ }^{1}$ Vaccination is vital not only in averting infections, it also mitigates the severity of disease and prevents some cancers (eg, cancers of the cervix and liver). ${ }^{2}$ The Expanded Programme on Immunisation (EPI), established in 1974 by the WHO, has greatly reduced the global burden of poliomyelitis, measles, tetanus, viral hepatitis B, diphtheria and other diseases. ${ }^{3}$ However, vaccination coverage remains low in 
many low and middle-income countries (LMICs). As a consequence, millions of children in such countries still die from diseases that could have been prevented with vaccines. ${ }^{2}$

Low immunisation coverage in LMICs has been attributed to several reasons, including family characteristics, parental attitudes and knowledge and inadequate information and communication. ${ }^{4}$ In particular, poor understanding of vaccines and vaccination schedules is associated with low immunisation coverage in LMICs. ${ }^{5}$ A randomised controlled trial has suggested that caregiver concerns regarding childhood vaccines may be due to conflicting information parents receive about the safety and risks of vaccines. ${ }^{6}$ Therefore, it is important that caregivers are directed to accurate information so that they can make informed decisions regarding vaccination of their child population. ${ }^{6}$

The use of messages that address caregivers' concerns and false beliefs may be an effective method for increasing compliance with vaccination schedules. Healthcare providers need strategies to successfully transfer vaccine-related information ${ }^{7}$ and to deal empathically and effectively with caregivers who have been exposed to antivaccination rumours and who question the need to vaccinate their children. ${ }^{8}$

Communication between and among providers and recipients of healthcare services has been highlighted as an emerging field of importance within the healthcare landscape. ${ }^{9}$ Active engagement and effective communication between healthcare providers and recipients are safe and efficient ways for improving a broad range of healthcare outcomes. ${ }^{10}$ Informing and educating caregivers about the benefits of vaccination could empower them to undertake effective preventive health care in general, which in turn could increase vaccination coverage. ${ }^{11}$ Therefore, it is important to identify relevant interventions for informing and educating caregivers about the importance of childhood vaccination in LMICs.

The purpose of this review is to assess the effects on vaccination coverage of interventions to inform or educate caregivers about childhood vaccination in low and middle-income countries, compared to standard immunisation practices.

\section{METHODS AND ANALYSIS}

The synopsis for this systematic review protocol is registered in the International Prospective Register of Systematic Reviews (http://www.crd.york.ac.uk/PROSPERO), ${ }^{12}$ registration number CRD42014010141. We will include randomised controlled trials (RCTs), with randomisation at either individual or cluster level. For cluster RCTs, we will only include those with at least two intervention and two control clusters.

In addition, we will include non-randomised controlled trials (non-RCTs), with allocation at either individual or cluster level. Non-RCTs are studies that allocated interventions by alternation between groups, by the use of birth dates or weekdays, or by other nonrandom methods. For cluster non-RCTs, we will only include those with at least two intervention and two control clusters. We will include only studies conducted in low and middle-income countries, as defined by the World Bank. ${ }^{13}$

This review will focus on interventions to inform or educate caregivers about the importance of vaccination. These interventions may include information sessions, group classes, oral presentations, slide shows, seminars, workshops, printed materials (pamphlets, posters and brochures), audio or video recordings and one-on-one education. These interventions can be delivered either face-to-face, by mail (email, letters or postcards), or through phone calls or mobile phone text messaging. Interventions aimed at reminding caregivers about vaccination sessions for their children, or recalling caregivers who have missed vaccination visits, are outside the scope of this review and will be excluded. We will compare the information on educational interventions to no intervention, standard immunisation practices in the study setting, alternative interventions or similar interventions implemented with different degrees of intensity.

The participants of interest will be caregivers (defined as parents, legal guardians or other persons assuming the parental role) to whom information or education about vaccination is given.

The primary outcome will be children's vaccination status (as defined by the trial authors). Secondary outcomes include caregivers' knowledge of vaccination, caregivers' intention to vaccinate their children, caregivers' satisfaction with the intervention and cost of the interventions.

We have developed a comprehensive search strategy for searching peer-reviewed and grey literature (see online supplementary appendix). Sources of peerreviewed literature to be searched include PubMed, Scopus, Cochrane Central Register of Controlled Trials (CENTRAL), ISI Web of Science (Science Citation Index), Cumulative Index of Nursing and Allied Health (CINAHL) and PDQ Evidence. In addition, we will search for ongoing trials in the WHO International Clinical Trials Registry Platform and Clinicaltrials.gov, and check reference lists of relevant reviews and full-text articles assessed for eligibility. We will include articles available by 31 May 2015 .

Two review authors will independently screen the search outputs for potentially eligible studies. Full texts of potentially eligible studies will be retrieved and the two authors will independently assess them for eligibility against the study inclusion criteria. We will endeavour to translate potentially eligible studies published in languages other than English and French. Disagreements about the inclusion of studies will be resolved through discussion and consensus. If disagreements are not resolved, a third author will arbitrate. Reasons for excluding potentially eligible studies will be provided. 
Two authors will independently extract data using a predesigned pilot-tested data collection form and compare their results, resolving discrepancies by consensus and arbitration by a third author, as required. The data to be extracted will include study design and methods, country setting (including income level as defined by the World Bank) and participant characteristics, intervention characteristics, study outcomes and study funding sources. In cases of missing or incomplete information in the included studies, we will contact study authors for further information.

In multicountry studies involving LMICs and also highincome countries, we will only extract data from LMICs. However, if data are not reported by country income levels we will contact the study authors to request separate data for LMICs. If, by the time the review is published, we have not yet received such data, we will classify the studies as awaiting assessment; and endeavour to update the systematic review as soon as such data become available.

The two authors will independently assess the risk of bias in each included study using the following criteria: adequacy of random sequence generation and allocation concealment (for risk of selection bias); blinding of participants and personnel (for risk of performance bias); blinding of outcome assessors (for risk of detection bias); completeness of outcome data (for risk of attrition bias); and completeness of outcome reporting (for risk of reporting bias). ${ }^{14}$ For each domain, we will classify the risk of bias as 'low' if the criterion was adequately addressed, 'unclear' if the information provided was not sufficient to make an informed judgement or 'high' if the criterion was not adequately addressed.

We will then summarise the assessments and categorise the included studies into three levels of bias: low, moderate and high risk of bias. Every study that is classified as low risk for all domains will be considered to be at low risk of bias. Any study that has a high risk of selection, detection or attrition bias, will be categorised as having a high risk of bias. All other studies will be considered to have a moderate risk of bias.

We will conduct data analysis using the latest version of the Cochrane Collaboration Review Manager statistical software (http://ims.cochrane.org/RevMan). Apart from cost of the intervention, the review outcomes are most likely to be reported as dichotomous data. We will express the results of each study as a risk ratio and its 95\% CIs (CI for dichotomous outcomes. ${ }^{14}$

We will pool the RRs and 95\% CIs of studies with identical outcomes and interventions; using random-effects meta-analysis, because of anticipated heterogeneity of study designs and participants. We will include data from eligible cluster RCTs in relevant meta-analyses after controlling for the design effect, using the intracluster correlation coefficient (ICC) derived from the same or a similar published cluster RCT. ${ }^{15} 16$

Statistical heterogeneity in each meta-analysis will be assessed using the $\chi^{2}$ test of homogeneity and quantified using the Higgins' $\mathrm{I}^{2}$ statistic. We will define statistical heterogeneity at the $10 \% \alpha$ level; and assess the source of observed statistical heterogeneity using subgroup analyses.

We will conduct subgroup analysis only for the primary outcome (ie, vaccination coverage), with subgroups defined by study design (RCTs vs non-RCTs) and type of intervention (information vs educational interventions). We have chosen each subgroup based on a specific hypothesis. Non-randomised studies are prone to selection bias and may over-estimate the efficacy of an intervention. Educational interventions (eg, structured and interactive communication tools) may lead to a better understanding of the importance of immunisation by caregivers and thus be more effective at increasing vaccination coverage than passive provision of information. ${ }^{17}$

We will perform a sensitivity analysis to determine the robustness of the findings to risk of bias (ie, including and excluding studies with a high risk of bias); with emphasis on allocation concealment, blinded outcome assessment and losses to follow-up (with a cut-off value of $25 \%$ ). ${ }^{18}$

We plan to use funnel plots to assess the possibility of publication bias across studies for every meta-analysis involving 10 or more studies. ${ }^{16}$ Publication bias leads to funnel plot asymmetry; but when there are fewer than 10 studies in a meta-analysis, funnel plot tests are unreliable in differentiating between real asymmetry and the play of chance. Other causes of funnel plot asymmetry include delayed-publication bias, location bias, selective outcome reporting, poor methodological design, inadequate analysis and fraud. ${ }^{16}$

We will use the GRADE approach to assess the certainty of the evidence for each outcome ${ }^{19}$ and present data in forest plots and 'Summary of Findings' tables. ${ }^{20}$ We have written this protocol following the recently released PRISMA-P guidelines, ${ }^{21}$ and will report the review according to the PRISMA statement; including any available revisions or extended guidance. ${ }^{22} 23$

\section{ETHICS AND DISSEMINATION}

We did not seek ethical approval for this study because the data to be collected cannot be linked to individuals. The findings of the review will make a significant contribution to the knowledge base of interventions for improving childhood vaccination coverage in low and middle-income countries. The study will gather evidence on how vaccination information or education impacts childhood vaccine uptake. We anticipate that this information will be useful to national and international stakeholders interested in improving the performance of childhood immunisation programmes in low and middle-income countries.

Twitter Follow Charles Wiysonge at @CharlesShey

Acknowledgements The authors would like to thank Alvina Matthee of the University of Stellenbosch's Faculty of Medicine and Health Sciences Library, for her assistance in developing the search strategies. The authors acknowledge the BMJ Open Editor and Lawrence Mbuagbaw for critical and insightful comments on an earlier version of this manuscript. 
Contributors LAL led the development of the protocol, wrote the first draft, coordinated and integrated comments from coauthors, approved the final version for publication and is the guarantor of the manuscript. NNM critically revised successive drafts of the manuscript, provided important intellectual input and approved the final version for publication. FBA critically revised successive drafts of the manuscript and approved the final version for publication. CSW conceived the study, provided supervision and mentorship to LAL, critically revised successive drafts of the manuscript, provided important intellectual input and approved the final version for publication.

Competing interests None declared.

Provenance and peer review Not commissioned; externally peer reviewed.

Open Access This is an Open Access article distributed in accordance with the Creative Commons Attribution Non Commercial (CC BY-NC 4.0) license, which permits others to distribute, remix, adapt, build upon this work noncommercially, and license their derivative works on different terms, provided the original work is properly cited and the use is non-commercial. See: http:// creativecommons.org/licenses/by-nc/4.0/

\section{REFERENCES}

1. Chauke-Moagi BE, Mumba M. New vaccine introduction in the East and Southern African sub-region of the WHO African Region in the context of GIVS and MDGs. Vaccine 2012;30:C3-8.

2. Oyo-Ita A, Wiysonge CS, Oringanje C, et al. Interventions for improving coverage of child immunization in low-and middle-income countries. Cochrane Database Syst Rev 2011;6:CD008145.

3. Levine OS, Bloom DE, Cherian T, et al. The future of immunisation policy, implementation, and financing. Lancet 2011;378:439-48.

4. Wiysonge CS, Uthman OA, Ndumbe PM, et al. Individual and contextual factors associated with low childhood immunisation coverage in sub-Saharan Africa: a multilevel analysis. PLOS ONE 2012;7:e37905.

5. Owais A, Hanif B, Siddiqui AR, et al. Does improving maternal knowledge of vaccines impact infant immunization rates? A community-based randomized-controlled trial in Karachi, Pakistan BMC Public Health 2011;11:239.

6. Williams SE, Rothman $\mathrm{RL}$, Offit $\mathrm{PA}$, et al. A randomized trial to increase acceptance of childhood vaccines by vaccine-hesitant parents: a pilot study. Acad Pediatr 2013;13:475-80.

7. Gowda C, Schaffer SE, Kopec K, et al. Pilot study on the effects of individually tailored education for MMR vaccine-hesitant parents on MMR vaccination intention. Hum Vaccin Immunother 2013;9:437-45

8. Burnett RJ, Larson HJ, Moloi MH, et al. Addressing public questioning and concerns about vaccination in South Africa: a guide for healthcare workers. Vaccine 2012;30:C72-8.
9. Willis N, Hill S, Kaufman J, et al. "Communicate to vaccinate": the development of a taxonomy of communication interventions to improve routine childhood vaccination. BMC Int Health Hum Rights 2013;13:23.

10. Coulter A, Ellins J. Effectiveness of strategies for informing, educating, and involving patients. BMJ 2007;335:24-7.

11. Williams $\mathrm{N}$, Woodward $\mathrm{H}$, Majeed $\mathrm{A}$, et al. Primary care strategies to improve childhood immunisation uptake in developed countries: systematic review. JRSM Short Rep 2011;2:81.

12. Lukusa LA, Adeniyi F, Wiysonge CS. Effects of interventions to inform or educate parents or caregivers about childhood vaccination in low and middle income countries. PROSPERO 2014: CRD42014010141. http://www.crd.york.ac.uk/PROSPERO REBRANDING/display_record.asp?ID=CRD42014010141.

13. The World Bank Group. Data. Countries and Economies. http://data.worldbank.org/country (accessed 16 May 2015).

14. Higgins JPT, Green S, eds. Cochrane Handbook for Systematic Reviews of Interventions, version 5.1.0 [updated March 2011]. The Cochrane Collaboration, 2011. Available from www.cochranehandbook.org (accessed 05 Jul 2015).

15. Kagina BM, Wiysonge CS, Machingaidze S, et al. The use of supplementary immunisation activities to improve uptake of current and future vaccines in low-income and middle-income countries: a systematic review protocol. BMJ Open 2014;4:e004429.

16. Bezuidenhout $H$, Wiysonge CS, Bentley JM. Risperidone for disruptive behaviour disorders in children with intellectual disabilities [Protocol]. Cochrane Database of Sys Rev 2012;(7):CD009988.

17. Wiysonge CS, Ngcobo NJ, Jeena PM, et al. Advances in childhood immunisation in South Africa where to now? Programme managers' views and evidence from systematic reviews. BMC Public Health 2012;12:578.

18. Nglazi MD, Bekker L, Wood R, et al. Mobile phone text messaging for promoting adherence to anti-tuberculosis treatment: a systematic review. BMC Infect Dis 2013;13:566.

19. Balshem $\mathrm{H}$, Helfand $\mathrm{M}$, Schünemann $\mathrm{HJ}$, et al. GRADE guidelines: 3 . Rating the quality of evidence. J Clin Epidemiol 2011;64:401-6.

20. Guyatt G, Oxman AD, Akl EA, et al. GRADE guidelines: 1. Introduction-GRADE evidence profiles and summary of findings tables. J Clin Epidemiol 2011;64:383-94.

21. Shamseer L, Moher D, Clarke M, et al, PRISMA-P Group. Preferred reporting items for systematic review and meta-analysis protocols (PRISMA-P) 2015: elaboration and explanation. BMJ 2015;349:g7647

22. Liberati A, Altman DG, Tetzlaff J, et al. The PRISMA statement for reporting systematic reviews and meta-analyses of studies that evaluate healthcare interventions: explanation and elaboration. BMJ 2009;339:b2700.

23. Welch V, Petticrew M, Tugwell $P$, et al, PRISMA-Equity Bellagio Group. PRISMA-Equity 2012 extension: reporting guidelines for systematic reviews with a focus on health equity. PLoS Med 2012;9: e1001333. 